\title{
On the Time Dependent Features of the Two-Colour Light Pulses Propagation in the Sodium Vapour
}

\author{
A.M. Alhasan and J. Fiutak \\ Institute of Physics, Faculty of Mathematics and Natural Sciences \\ Pomeranian Pedagogical University in Słupsk \\ Arciszewskiego 22b, 76-200 Słupsk, Poland
}

(Received June 29, 2005; revised version October 4, 2005)

\begin{abstract}
The phase and group velocities of the pulsed light are suitably defined and calculated. So are the duration of both pulses, probe and coupling, and their energies as a function of the travelled distance. The time evolution, for a given distance, of the dressed atom state is described by the Liouville-von Neumann equation for the density matrix.
\end{abstract}

PACS numbers: 42.50.Md, 42.65.--k, 42.50.Hz

\section{Introduction}

The development of the electromagnetically induced transparency (EIT) technique, reviewed by Harris [1], resulted in the slowing down of the light [2-4] and the storage of optical information [5-7]. Both these effects, slow light and light storage, showed a good relation to the quantum interference effect [8 and references therein]. The group velocity reduction has been considered in different connections. A method based on the steady state solution showed the mechanism for the loss of the probe field [9]. The slow light propagation in an open three level system has been analyzed numerically in [10]. Kasapi et al. [11] have described the group velocity reduction associated with the propagation dynamics for EIT pulses in ${ }^{208} \mathrm{~Pb}$ vapour. Furthermore, Greentree et al. [12] have demonstrated the influence of turn-on and turn-off transients on the EIT in ${ }^{87} \mathrm{Rb}$ magneto-optical trap. Roberts et al. [13] have calculated the ground state collisional decay rate that limits delay and storage times in EIT experiments with condensates. The nonlinear process associated with the group velocity reduction, such as pulse compression, has been demonstrated by Harris and Hau [14]. The soliton behaviour in three-level system has been discussed in [15, 16 and reference therein]. It has been 
demonstrated that the three-level atom soliton can propagate with the velocity of light [15]. The conditions for subluminal to superluminal propagation through the phase control of the group velocity in a $\mathrm{V}$ shaped three-level system have been discussed in [17]. Finally, the propagation through an anomalous dispersion medium showed the pulse propagation at a negative group velocity $[18,19]$.

The standard description of the EIT and its related phenomena, i.e. the pulse delay, advancement and the light storage, is based on the steady state approach. Therefore the pulse shape and its duration cannot be properly taken into account. Moreover, it is assumed that the refractive index and the absorption length are determined for the constant field. Thus, the change during the propagation of the pulse shape and its intensity are usually neglected, which is obviously not justified. For this reason we propose, in the second section, the definitions of the group velocity for the light pulse and the parameters characterizing its shape. So we are able to take into account not only the initial time dependence of the light intensity but also the change of the pulse in the course of the propagation. As an example of the proposed approach, we consider the propagation of two-colour pulse tuned to the $S_{1 / 2}-P_{1 / 2}$ transition with the lower $S_{1 / 2}$ and upper $P_{1 / 2}$ levels split by hyperfine interaction. In the third section we describe our atomic system, its state evolution ruled by the Liouville-von Neumann equations for the density matrix and the propagation of the light pulses described by the reduced Maxwell equations. Finally, in the fourth section we represent the numerical results for the pulse and atoms characterizations as functions of time and distance. We consider the long and short pulse durations as compared with the spontaneous decay time. In all cases we have found that the group velocity and other features do depend on the distance that the pulse travels.

\section{The characteristics of the light pulse}

The light pulse is generally, represented at a given time $t$ and a distance $z$ by the electric field

$$
E(z, t)=\exp \left(-\mathrm{i} \omega_{0}\left(t-\frac{z}{c}\right)\right) V\left(z, t-\frac{z}{c}\right)+\text { c.c. },
$$

where $c$ stands for the velocity of light in vacuum. In the following we will use also the Fourier transform $\tilde{V}$ of the shape function

$$
\tilde{V}(z, \omega)=\frac{1}{2 \pi} \int \exp \left(-\mathrm{i}\left(\omega-\omega_{0}\right) t^{\prime}\right) V\left(z, t^{\prime}\right) \mathrm{d} t^{\prime},
$$

where $t^{\prime}=t-z / c$ is the retarded time. $\tilde{V}(z, \omega)$ is usually related to the initial $\tilde{V}(0, \omega)$ by

$$
\tilde{V}(z, \omega)=\exp \left(-\mathrm{i}(n-1) \omega \frac{z}{c}\right) \tilde{V}(0, \omega) .
$$

The last relation may be thought as a definition of the complex refractive index $n$, which is, in general, a function of $\omega$ and $z$. In the following, we will use both the functions $V(z, t)$ and $\tilde{V}(z, \omega)$ to define the mean $\bar{O}$ of any observable $O$ in the same manner we do in quantum mechanics, i.e. 
or

$$
\langle O\rangle=\frac{\int V^{*}(z, t) \hat{O}_{t} V(z, t) \mathrm{d} t}{\int|V(z, t)|^{2} \mathrm{~d} t}
$$

$$
\langle O\rangle=\frac{\int \tilde{V}^{*}(z, \omega) \hat{O}_{\omega} \tilde{V}(z, \omega) \mathrm{d} \omega}{\int|V(z, \omega)|^{2} \mathrm{~d} \omega},
$$

where the operators $\hat{O}_{t}$ and $\hat{O}_{\omega}$ are in the time and the frequency representation, respectively. So the mean pulse time is given by

or

$$
T(z)=\frac{\int V^{*}(z, t) t^{\prime} V\left(z, t^{\prime}\right) \mathrm{d} t^{\prime}}{\int\left|V\left(z, t^{\prime}\right)\right|^{2} \mathrm{~d} t^{\prime}}+\frac{z}{c}
$$

$$
T(z)=\frac{-\mathrm{i} \int \tilde{V}^{*}(z, \omega) \frac{\partial \tilde{V}(z, \omega)}{\partial \omega} \mathrm{d} \omega}{\int|V(z, \omega)|^{2} \mathrm{~d} \omega}+\frac{z}{c}=\frac{z}{c} \operatorname{Re}\left\langle\left(n+\omega \frac{\mathrm{d} n}{\mathrm{~d} \omega}\right)\right\rangle .
$$

In fact, the time $T(z)$ is the time required for transport the pulse energy averaged over the pulse shape. For the symmetrical pulse it is identical with the time of the pulse maximum propagation. Furthermore, our definition is more convenient for the theoretical computations.

Thus the group velocity $\bar{v}_{\text {g }}$ of the light averaged over the distance $z$,

$$
\bar{v}_{\mathrm{g}}=\frac{z}{T(z)-T(0)},
$$

is also given as

$$
\bar{v}_{\mathrm{g}}=\frac{c}{\operatorname{Re}\left\langle\left(n+\omega \frac{\mathrm{d} n}{\mathrm{~d} \omega}\right)\right\rangle} .
$$

The above formula differs from the one most often used in the literature in which we average the denominator over the pulse shape instead of taking its value at $\omega=\omega_{0}$. We can also define the group velocity at a given distance $z$ as

$$
v_{\mathrm{g}}=\left(\frac{\mathrm{d} T}{\mathrm{~d} z}\right)^{-1} \text {. }
$$

However, we must have in mind that in the experiment we really measure the transit time $T(L)$ and thus the group velocity averaged over the length $L$ of the medium. Therefore, the definition (8) of the averaged group velocity is more significant for the theoretical analysis of the pulse change in the course of the propagation. The mean frequency $\langle\omega\rangle$ can be calculated from the formula (5) or, since $\omega-\omega_{0} \rightarrow \mathrm{i} \frac{\partial}{\partial t}$, as the average of the time derivative of the pulse phase $\varphi(z, t)$, i.e.

$$
\langle\omega\rangle=\left\langle\frac{\partial \varphi(z, t)}{\partial t}\right\rangle+\omega_{0}
$$

The frequency $\omega_{0}$ is often assumed to be the value of $\langle\omega\rangle$ at $z=0$. The phase velocity we shall define as

$$
v_{\mathrm{p}}=\langle\omega\rangle /\langle k\rangle
$$


with the mean value of the wave vector given by

$$
\langle k\rangle=-\frac{\partial\langle\varphi\rangle}{\partial z}+\frac{\omega_{0}}{c} .
$$

The characteristic duration time of the pulse is defined as

$$
\Delta T=\left(\left\langle t^{\prime 2}\right\rangle-\left\langle t^{\prime}\right\rangle^{2}\right)^{1 / 2}=\left[\left\langle\left(t-\frac{z}{c}\right)^{2}\right\rangle-\left(\left\langle t-\frac{z}{c}\right\rangle\right)^{2}\right]^{1 / 2} .
$$

The dispersion of the frequency,

$$
\Delta \omega=\sqrt{\left\langle\omega^{2}\right\rangle-\langle\omega\rangle^{2}},
$$

is related to $\Delta T$ by the uncertainty relation

$$
\Delta \omega \Delta T \geq 1 / 2 \text {. }
$$

We may often assume that $\Delta \omega \cong 1 /(2 \Delta T)$ since the pulse shape is approximately Gaussian. When the frequency of the light is well tuned to the atomic transition frequency, we may be tempted to use the complex refractive index $n$ at the line centre instead of the frequency averaging in formula (9). This seems to be well justified in the limit of strong power broadening, i.e. when the Rabi frequency at the pulse peak $\Omega_{\max }$ satisfies the condition $\Omega^{2} \gg \gamma \gamma_{1,2}$ where $\gamma$ is the spontaneous decay constant and $\gamma_{1,2}$ gives the relaxation rate of the Raman coherence $\rho_{1,2}$ component of the density matrix. In our calculations we have assumed $\gamma_{1,2}$ equal to $10^{-5} \gamma$, so that the frequency dependence of the refractive index is rather weak. Unfortunately, as we shall see later on, this picture is sometimes oversimplified as it does not account for all nonlinear effects in the two pulses mutual interaction and the effect following the finite pulse time, i.e. the absorption on the front of the pulse and the free induction decay. Moreover, it is customary to consider the refractive index $n(\omega)$ and its derivative as a function of the probe pulse frequency $\omega_{\mathrm{p}}$, which is not justified when the nonlinear effects are important.

\section{The atomic state description and the pulse propagation}

In the recent paper [20] we have described the quantum interference effect for the sodium $D_{1}$ line with the hyperfine structure shown in Fig. 1. Furthermore, we have investigated the steady state for the same system [21]. In the present paper we are going to describe the propagation of both the coupling field and the probe beams properly tuned so that their frequencies $\omega_{\mathrm{c}}$ and $\omega_{\mathrm{p}}$ are bound to the condition $\omega_{\mathrm{p}}-\omega_{\mathrm{c}}=\omega_{2,1}$, which is usually assumed in the EIT investigations. The two pulses in question, probe and coupling, are assumed to travel together. Moreover, in most cases we add with some delay the second coupling pulse so that the restoring of the probe pulse can be checked. According to the standard procedure, we shall describe the dressed atom state using the density matrix Liouville--von Neumann equations as in [20]. However, these equations will be modified to account for the two-colour excitation by the coupling and probe beams in the similar manner to that used in the paper of Zhu [22]. So, the interaction of the atom with light has to be replaced by the two components with the frequency $\omega_{\mathrm{c}}$ and $\omega_{\mathrm{p}}$, 


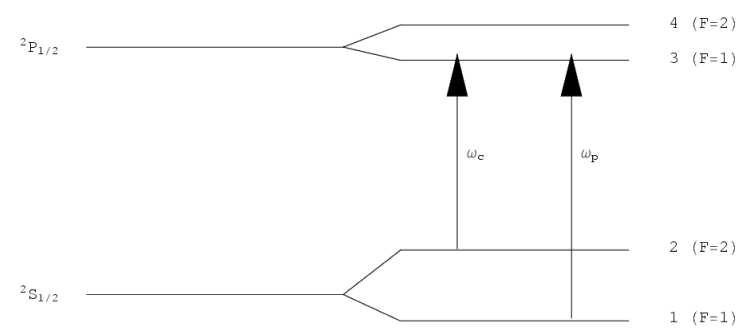

Fig. 1. The energy level diagram of the sodium atom. $\omega_{\mathrm{p}}$ and $\omega_{\mathrm{c}}$ stand for the probe and coupling fields frequencies, respectively. $\omega_{2,1}=1.772 \times 10^{9} 2 \pi \mathrm{s}^{-1}, \omega_{4,3}=189 \times 10^{6} 2 \pi \mathrm{s}^{-1}$ and the spontaneous decay rate $\gamma=6.25 \times 10^{7} \mathrm{~s}^{-1}$.

respectively. To simplify the description, we assume the coupling beam is exactly tuned to the atomic transition frequency $2 \rightarrow 3$ while the probe beam is tuned to the $1 \rightarrow 3$ transition except the case (b) in Fig. 3, in which both are detuned by the same amount. The atomic states are described by the same twenty eight components of the density matrix as in the papers [21] and [23]. The most relevant are: the populations $n_{i}$ of all the four levels, two complex Hertzian (optical) coherences $\rho_{1,3}$ and $\rho_{2,3}$, and the Raman coherence $\rho_{1,2}$, which is responsible for the coherent mixture of the lower states meant for the nonlinear effects in EIT, light slowing down and restoring.

As far as the propagation is concerned, the relevant equations are the reduced Maxwell equations for the radiation fields. We assume that the propagation of the probe and coupling pulses can be described by separated equations, i.e.

$$
\frac{\partial V_{\mathrm{p}}}{\partial z}=A_{\mathrm{p}}\left(\rho_{1,3}-\sqrt{5} \rho_{1,4}\right)
$$

and

$$
\frac{\partial V_{\mathrm{c}}}{\partial z}=A_{\mathrm{c}}\left(\rho_{2,3}-\rho_{2,4}\right)
$$

where the parameters $A_{\mathrm{p}}, A_{\mathrm{c}}$ depend on the atomic density and the transition frequencies. The combined optical coherences on the right hand side of (17) and (18) are proportional to the dipole moment for the transition frequency $1 \rightarrow 3,4$ and $2 \rightarrow 3,4$. We shall make use of the relative units in which the frequencies and the relaxation rates are related to the spontaneous decay. So we shall replace the pulse shape functions $V_{\mathrm{p}}$ and $V_{\mathrm{c}}$ by the relative Rabi frequencies $\Omega_{\mathrm{p}}$ and $\Omega_{\mathrm{c}}$. The distance $z$ will be normalized to the Beer absorption length for the probe in the weak field limit, which is of the order of $10^{-4} \mathrm{~cm}$ in a typical experiment.

\section{The numerical results}

The aim of our numerical calculations is to explore the physical mechanism of the various phenomena observed when the two-colour pulsed light is travelling through the sodium vapour. Attention is paid to the space dependence of the group velocity, the light storage and the delay or the advancement of the pulsed light. Our goal is to describe the propagation of co-propagating fields without the 


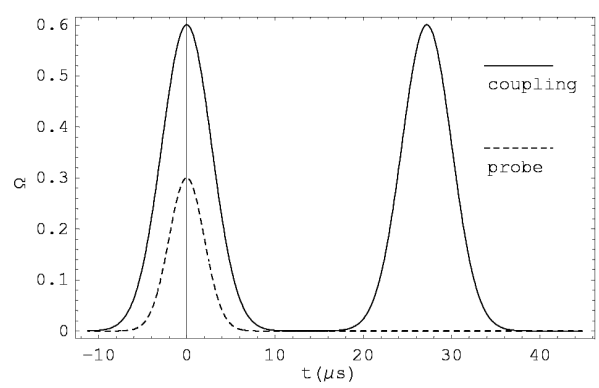

Fig. 2. The shape functions of the probe pulse and coupling fields at $z=0$ for long pulses.

commonly used adiabatic or the perturbation theory. We do not attempt to refer to any specific experiments, although we assume that the probe field (p) and the coupling field (c) are tuned as shown in Fig. 1. In the following we shall assume the Gaussian shape of both pulses but with cut off the far wings, as shown in Fig. 2. We consider some situations with initially Gaussian pulses but for different duration times measured by $\Delta T_{\mathrm{p}}$ and $\Delta T_{\mathrm{c}}$. The first situation, as shown in Fig. 2 , is devoted to pulses which are assumed to be long as compared with the spontaneous decay time $\gamma^{-1}$, while the second one is short, i.e., a nanosecond pulse. In all situations we analyse the pulsed light impact on the state of the atom at $z=0$. Particularly, we calculate the populations, the dipole moments and the Raman coherence. We may thus try to predict the change of the light pulse shape, its absorption and the light storage effect, at least for small distances. In the following, we show the pulse energy variation in the course of propagation as well as the mean pulse time, and its duration characterizations.

\subsection{On and off resonant long pulses propagation}

In Figs. 3 to 6 we represent the results for long pulse (see Fig. 2), subdivided into case (a), with both pulses tuned exactly as in Fig. 1, and case (b) with the frequencies of both pulses shifted by $10 \gamma$ (hereafter, the terms: case (a) and/or case (b) will allways refer to these definitions). In both cases only the first level is initially populated, since we have assumed the low temperature limit.

4.1.1. The impact of the atom at $z=0$ for long pulses in connection to the delay or the advancement, light storage, and EIT effect

The imaginary part of the dipole moment, as shown in Fig. 3 at $z=0$, is a good indication of the subsequent change of the propagating pulse shape. So we may expect, in the case (a), that the front of the probe field is absorbed, while later on, for $t>0$, this field is enhanced. The coupling field behaves in the opposite way as a result of the initial absence of the population $n_{2}$ and the coherent coupling of both pulses, characteristic of EIT. The net results are the high transparency and delay of the probe field combined with the advancement of the coupling pulse. In the case (b), the EIT effect is weaker, so that the absorption of the probe field is 

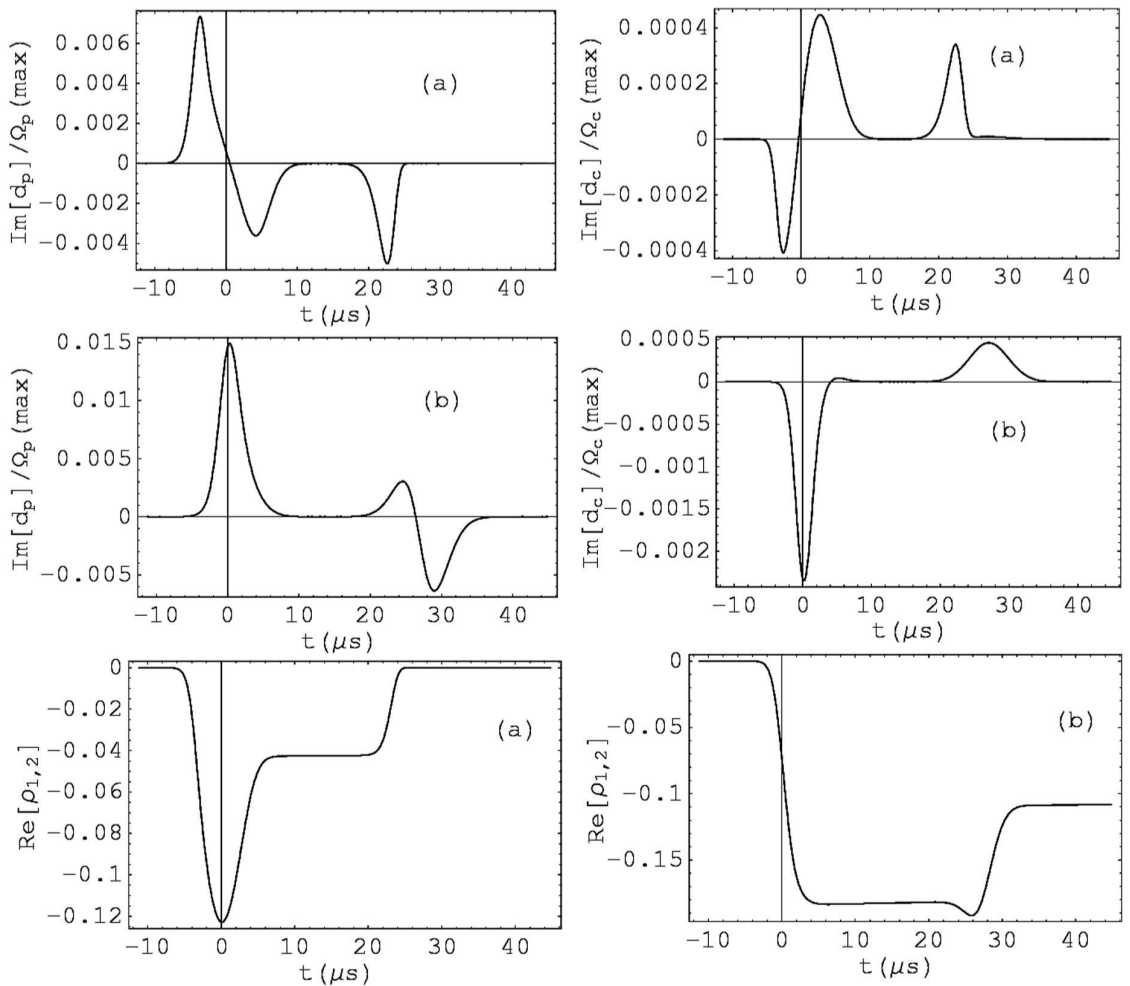

Fig. 3. The imaginary part of the dipole moment for the probe $d_{\mathrm{p}}$ and coupling $d_{\mathrm{c}}$ fields, the real part of the Raman coherence $\rho_{1,2}$ at $z=0$. Case (a) - the detuning $\Delta_{\mathrm{p}}=\Delta_{\mathrm{c}}=0$ and case $(\mathrm{b})-\Delta_{\mathrm{p}}=\Delta_{\mathrm{c}}=10 \gamma$.

much stronger than in the previous case. It is rather an unusual effect of increased absorption for the off resonance light frequency. In both cases the restored field is created at the expense of the second coupling pulse due to the Raman coherence. The Raman coherence for the case (a) in Fig. 3, shows a maximum at $t=0$, i.e., for the maximal values of both pulses intensities, and reaches the value -0.04 after the pulses are switched off. In the case (b) the Raman coherence increases in time up to the value -0.175 after the pulse. This means that the coherent interference effects for both pulses, and therefore the creation of the restored field, are more intense than in the case of exactly tuned fields. Quite obviously, the Raman coherence is reduced after the second pulse is switched on, so that the restored field is created.

\subsubsection{The space dependence of energy and light storing}

The electromagnetically induced transparency is demonstrated in Fig. 4, where the probe field intensity, in the case (a), slowly decreases with the distance, while the coupling field energy rather increases for $z>50$. The restored pulse gains the energy at the expense of the second coupling pulse, reaching maximum 

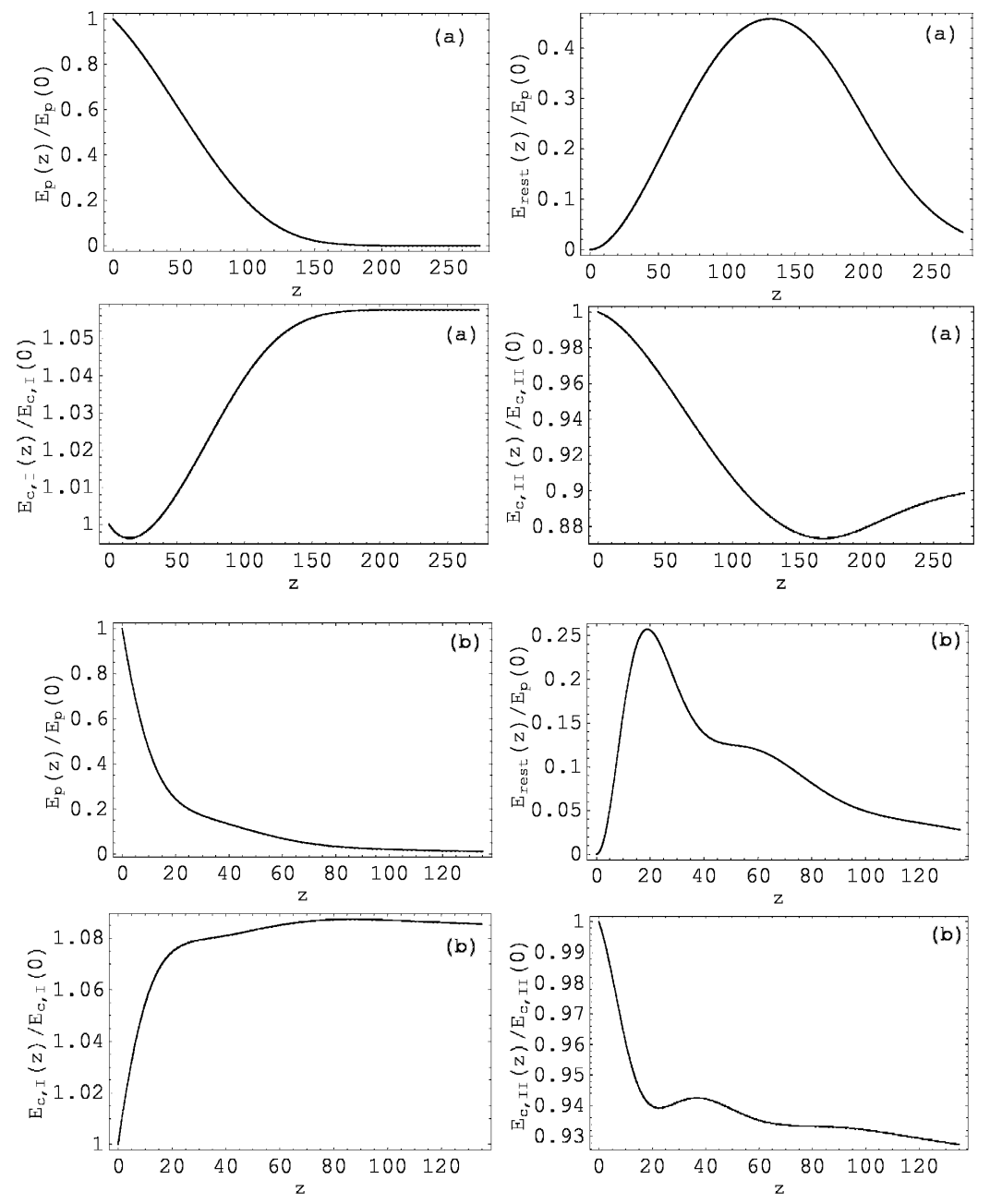

Fig. 4. The relative energies for the probe $E_{\mathrm{p}}(z)$, the first coupling $E_{\mathrm{c}, \mathrm{I}}$, the second coupling $E_{\mathrm{c}, \mathrm{II}}$, and the restoring field $E_{\text {rest }}$ as a function of $z$ for the cases (a) and (b). The distance is measured in terms of the Beer length.

for relatively long distance. The unexpected effect is that, for long distance, the restored field might be much more intense than the probe field. In the case (b), shown in Fig. 4b, the detuning of both pulses results in the stronger absorption of the probe field and fast increase in the coupling field intensity. The maximum of the restored field is also reached at relatively small distance $z \approx 20$, and its intensity is smaller than in the previous case. The obvious explanation is the detuning of the second coupling pulse.

\subsubsection{The space dependence of delay and group velocity for long pulses}

The time delay of the probe field, as shown in Fig. 5, for the case (a), is quite remarkable. The coupling field is, in this case, initially advanced, reaching 

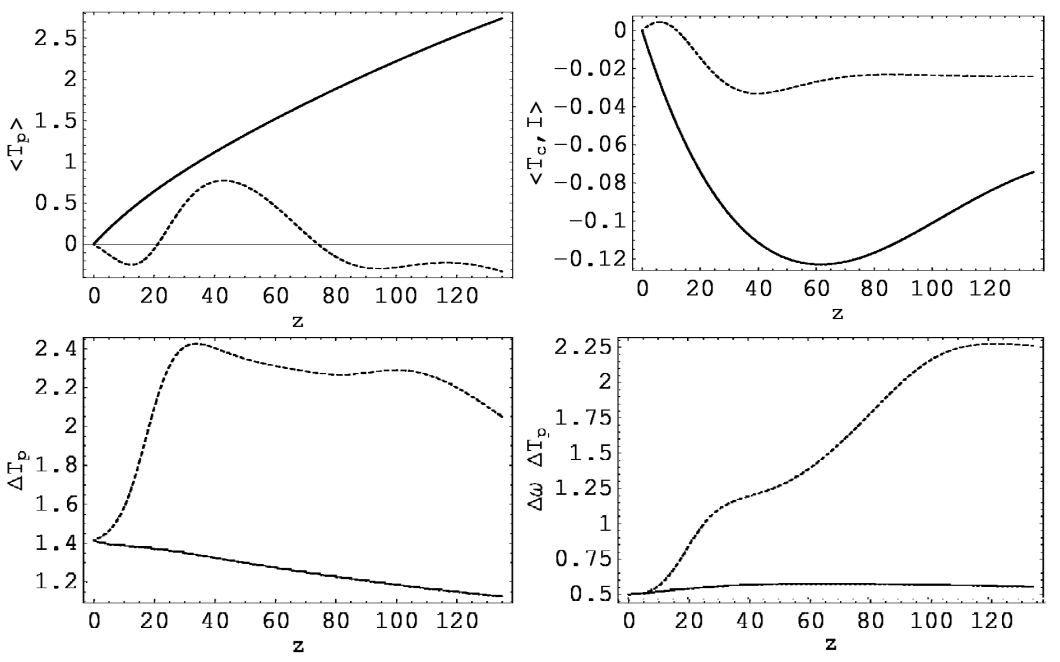

Fig. 5. The delay or the advancement of the probe field $\left\langle T_{\mathrm{p}}\right\rangle$ and the first coupling field $\left\langle T_{\mathrm{c}}\right\rangle$, the width $\Delta T_{\mathrm{p}}$ and the uncertainty product for the probe field in the course of propagation. The continuous line is for the case (a) and the dashed one is for the case (b). The time is measured in $\mu$ seconds.

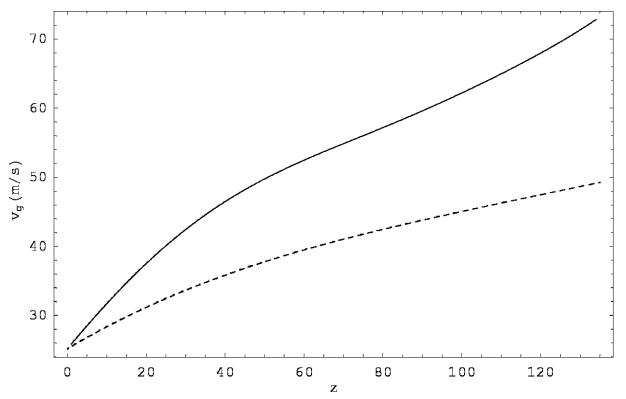

Fig. 6. The group velocity as a function of $z$ for the case (a). The continuous line for the smooth relation (10) and the dashed line for the space averaged velocity, relation (8).

its maximum for $z \cong 50$. In both cases the group velocity depends on the distance. In the case (b), our calculation indicates that the probe field is slightly advanced for small $z$, although in the most important region the probe pulse is delayed. The coupling field is slightly advanced. In this case the group velocity is strongly dependent on $z$. The probe pulse shape remains Gaussian for the case (a), as it follows from the unusually small deviation of the uncertainty product $\Delta T_{\mathrm{p}} \Delta \omega_{\mathrm{p}}$ from the one half value. The width of the pulse decreases with $z$. On the contrary, in the case (b) the probe pulse changes its shape in the course of propagation. It seems worth stressing that the dependence of the group velocity on the distance 
$z$ cannot be neglected even for relatively smooth delay of the probe field in the course of the propagation (see Fig. 6 for the case (a)). Thus, we may expect that the experimentally measured group velocity depends on the length $L$, that the pulse has to travel.

\subsection{The short pulse propagation}

As for some examples of the short pulse, we choose the initially Gaussian pulses presented in Fig. 7a and relatively weaker than the former one as depicted in Fig. 7b.
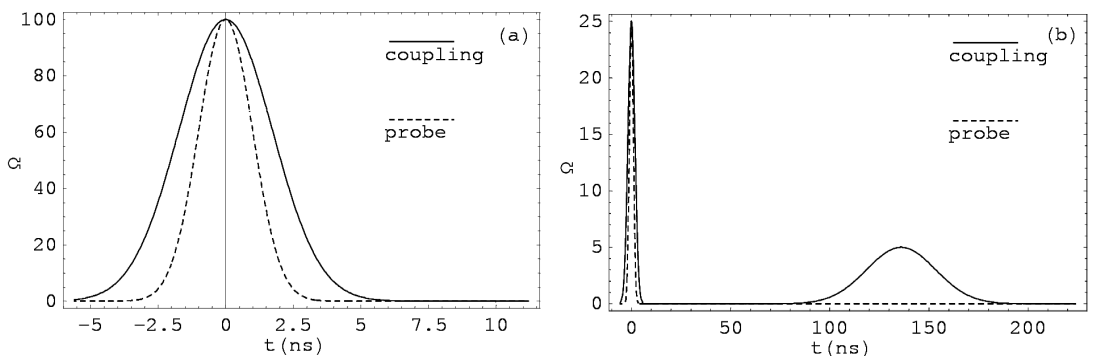

Fig. 7. The shape functions of the probe pulse and coupling field at $z=0$ for short duration pulses. Time is measured in nanoseconds. On the left part (a) we represent the strong field case. On the right part (b) we represent a less intense situation. A delayed component to the coupling field is added to check the restoring field.

\subsubsection{Intense pulse delays with splitting}

For intense pulses, the dependence of the populations on time (Fig. 8) shows that the atom behaves like a three-level system, i.e., both the third and the fourth
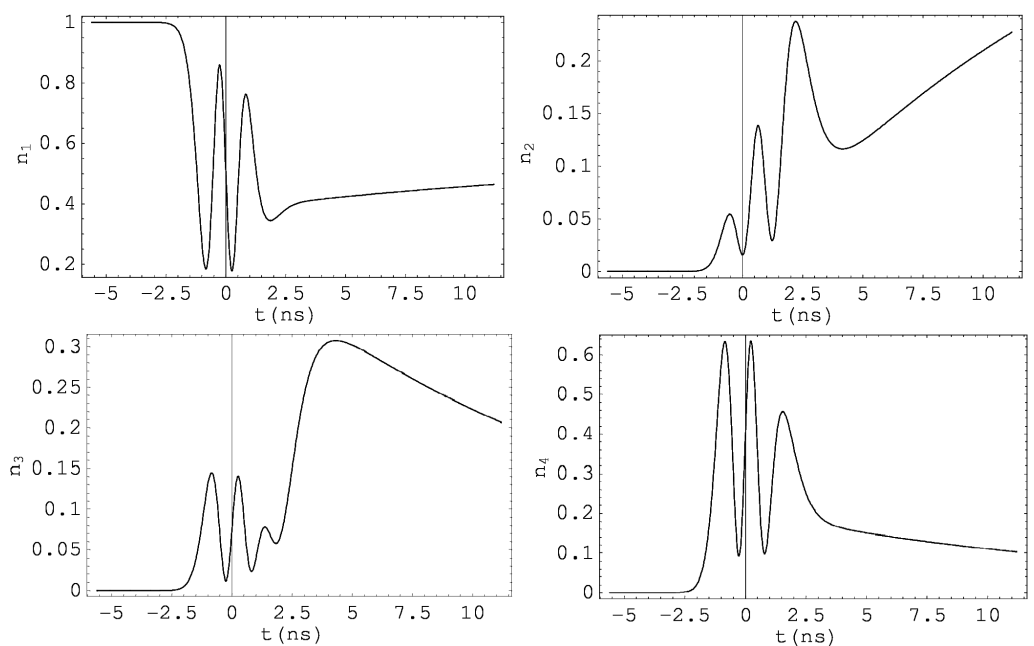

Fig. 8. The population of the hf levels at $z=0$ for strong nanosecond pulses shown in Fig. $7 \mathrm{a}, n_{1}\left(t_{0}\right)=1$. 
levels are coupled to the ground state levels by the probe and coupling fields. Moreover, the combined pulse, probe and coupling together, behaves like a pulse with the initial area above the $4 \pi$ value. We have reached this conclusion after comparing our results with those presented in paper [24], where the state of the atom excited by the pulses with various area tuned to the $S_{1 / 2}-P_{1 / 2}$ transition has been analysed. We are not able, however, to define the field area in clear fashion for the two-colour pulses. We may only estimate roughly the probe pulse area as $5 \pi$. The absorption in the front of the probe pulse and the free induction decay after the pulse result in the delay are shown in Fig. 9. The delay and change in the pulse shape for the coupling field are very small. However, the uncertainty product for the probe field increases, since the pulse is split into two parts. The pulse is quite justifiably defined for the probe field, at a given $z$ and decreases when the pulse propagate. The transparency of both pulses is extremely high, as shown in Fig. 9. The energy of the coupling pulse increases in the course of the propagation. Quite obviously, the EIT must play an important role in this case, since the transparency is much more conspicuous than in the case of single
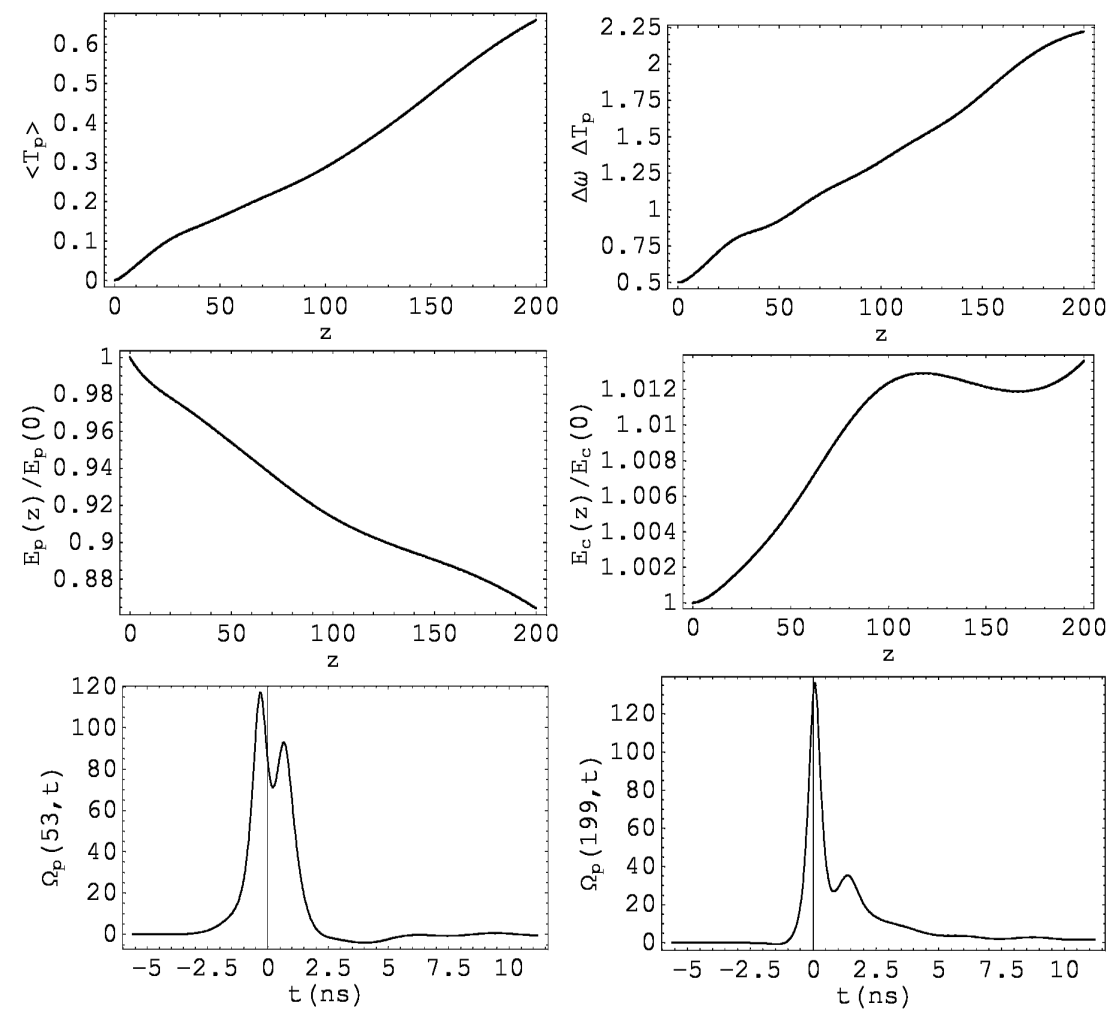

Fig. 9. The delay, the uncertainty product, the relative energies for probe and the first coupling field, and the shape functions for probe pulse at $z=53, z=199$. Time is measured in nanoseconds. Case (a) of Fig. 7 is considered. 
pulse with $4 \pi$ area (compared with the results in [24]). The splitting of the probe pulse is shown in Fig. 9, for $z=53$, while, for $z=199$, one of the split pulse components is fading away. Apparently, the $4 \pi$ is going to transfer into $2 \pi$ pulse. The coupling field is practically unchanged. We have found that the populations for $z=199$ oscillate like $4 \pi$ pulses. So, we may conclude that the combined probe and coupling field behave like the $5 \pi$ (at $z=0$ ) pulse in the work of Miklaszewski and Fiutak [24], but in a remarkably more stable way. On the other hand, some caution is required since the field area cannot be precisely defined.

\subsubsection{Short pulse propagation with light storage}

The pulses presented in Fig. $7 \mathrm{~b}$ are as short as the previous ones but less intense. The second coupling pulse is added to test the probe pulse restoring effect. The populations at $z=0$, given in Fig. 10, are reshaped during the pulse, by the absorption of the front part of the probe field and the subsequent induced emission of the coupling field. Later on, after the pulse, the spontaneous emission dominates until the second coupling pulse is switched on. The electric dipole moments corresponding to the probe field $\left(d_{\mathrm{p}}\right)$ and the coupling field $\left(d_{\mathrm{c}}\right)$ are oscillating with the frequency $\Delta \omega_{\mathrm{u}}$, as shown in Fig. 10. Their imaginary parts
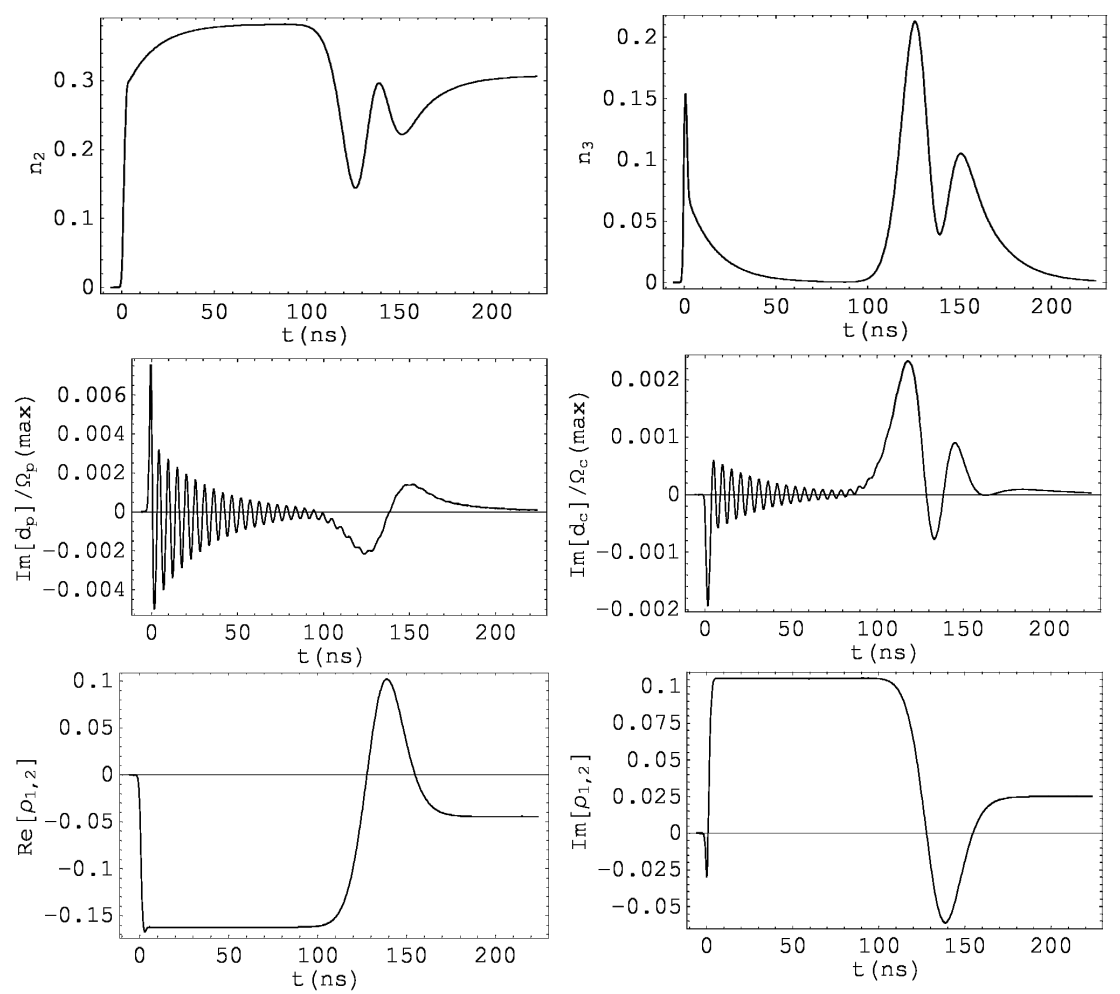

Fig. 10. The populations, the imaginary part of the dipole moment, and the Raman coherence at $z=0$ for the shape pulses presented in Fig. $7 \mathrm{~b}, n_{1}\left(t_{0}\right)=1$. 

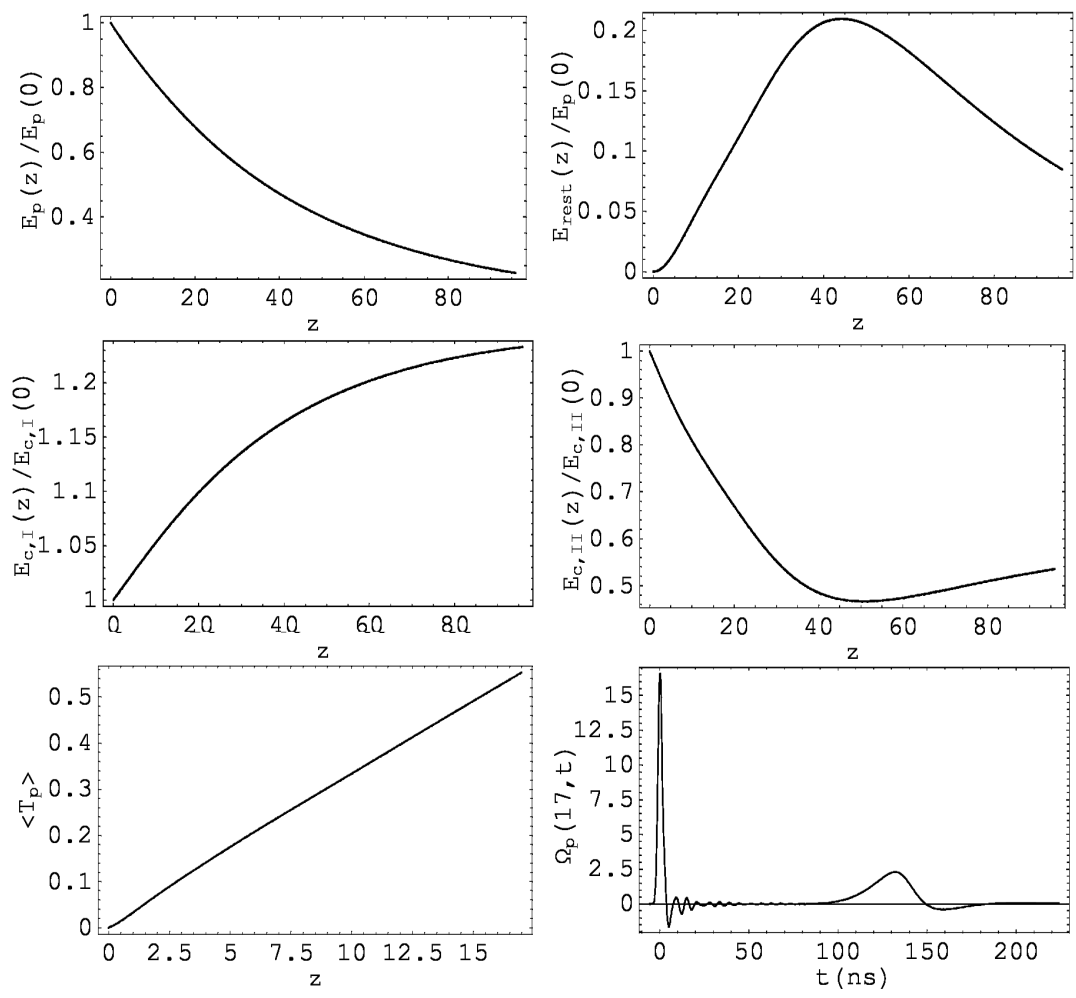

Fig. 11. The space dependence of the relative energies of the pulses as well as the delay time of the probe pulse and its shape at $z=17$. The time is measured in nanoseconds.

indicate that the absorption of the probe field and the second coupling pulse are quite big, while the first coupling pulse increases its energy with the distance at the expense of the probe field. The Raman coherence presented in Fig. 10 is quite big, so we may expect the strong field restoring effect. The strong absorption at the front results in the delay of the probe pulse (see Fig. 11) and in its broadening. The shape of the coupling field does not change appreciably and the delay time is small. Although, the shape of the probe field is changed, the deviation from the initial Gaussian shape is small. The energy changes with the distance, presented in Fig. 11, are rather smooth. The decrease in the probe pulse energy is accompanied by the increase in the coupling pulse energy, so that the total energy of both pulses decreases quite slowly. The pulse shape at $z=17$, presented in Fig. 11, remains nearly unchanged for both pulses, except the restored pulse, which is created at the expense of the second coupling pulse.

\section{Conclusions}

The definitions of the important characteristics of the pulse proposed in the present paper have been proved to be very useful. We have particularly in mind 
the group velocity, the mean deviation of the pulse time $\Delta T$, and of frequency $\Delta \omega$. We have shown that the group velocity, defined as the ratio of the medium length $L$ to the transit time $T(L)$, is related to the refraction index in the standard way, except that the average over the pulse is taken into account. In spite of that, our results differ meaningfully from the usual description, which does not account properly for the time and distance dependence of the light pulse. Particularly, the dependence of the pulse duration is proved to be very important for all features of the light propagation. The transit time is, as a rule, of the same order as the pulse duration. This is to be expected, since the distortion of both pulses results from their cooperation when they overlap. More precisely, the pulse is modified either by absorption or by the induced Raman scattering, controlled by the Raman coherence $\rho_{1,2}$, and the induced emission without the EIT effect. The best example of the Raman scattering action is the coincidence of the populations and the corresponding Raman coherence $\rho_{1,2}$. For the nanosecond pulses, the stimulated Raman and induced emissions are important. In fact, the time delay of the $2 n \pi$ pulses is possible for a single monochromatic pulse (cf. [24-26]) and so is the prolonged propagation length. However, the cooperation of both pulses seems to enhance appreciably both effects. The successive result, which seems to be of the same importance, is the nonlinear dependence of the transit time on the propagation length (see Figs. 5, 9,11) that is why the group velocity depends on $z$ (Fig. 6). This dependence shows a transition from superluminal propagation, where the group velocity is a negative finite or infinite, to subluminal (see Fig. 5).

As far as the storage of light is concerned, we have shown that the restoring field for short pulses is created mainly at the expense of the second coupling field.

Coming to the end of our discussions, let us turn our attention to the uncertainty product $\Delta \omega \Delta T$, which is equal one half for the Gaussian pulse. The results shown in Fig. 5 confirm the commonly accepted opinion that deviations from the Gaussian shape are small, except for long distances, where the pulse is very weak. The strong deviation from the Gaussian type for nanosecond pulse (Fig. 9) follows from the splitting of the $4 \pi$ pulse into two $2 \pi$ pulses.

\section{Acknowledgment}

The authors are grateful to the Pomeranian Pedagogical University in Słupsk for financial support. The National Laboratory FAMO (UMK) in Torun is acknowledged for partial financial support. A.M. Alhasan acknowledges Dr. Radek Szmytkowski for reading the manuscript. 


\section{References}

[1] S.E. Harris, Phys. Today 50, 36 (1997).

[2] L.V. Hau, S.E. Harris, Z. Dutton, C.H. Behroozi, Nature 397, 594 (1999).

[3] J.E. Heebner, R.W. Boyd, J. Mod. Opt. 49, 2629 (2002).

[4] M.S. Bigelow, N.N. Lepeshkin, R.W. Boyd, Phys. Rev. Lett. 90, 113903 (2003).

[5] C. Liu, Z. Dutton, C.H. Behroozi, L.V. Hau, Nature 409, 490 (2001).

[6] D.F. Phillips, A. Fleischhauer, A. Mair, R.L. Walsworth, M.D. Lukin, Phys. Rev. Lett. 86, 783 (2001).

[7] A.B. Matsko, Y.V. Rostovtsev, O. Kocharovskaya, A.S. Zibrov, M.O. Scully, Phys. Rev. A 64, 043809 (2001).

[8] Z. Ficek, S. Swain, J. Mod. Opt. 49, 3 (2002).

[9] L. Deng, M.G. Payne, E.W. Hagly, Opt. Commun. 198, 129 (2001).

[10] E. Cerboneschi, F. Renzoni, E. Arimondo, arXiv:quant-ph/0102045 (2001).

[11] A. Kasapi, J. Maneesh, G.Y. Yin, S.E. Harris, Phys. Rev. Lett. 74, 2447 (1995).

[12] A.D. Greentree, T.B. Smith, S.R. de Echaniz, A.V. Durrant, J.P. Marangos, D.M. Segal, J.A. Vaccaro, Phys. Rev. A 65, 053802 (2002).

[13] D.C. Roberts, T. Gasenzer, K. Burnett, Phys. Rev. A 66, 023801 (2002).

[14] S.E. Harris, L.V. Hau, Phys. Rev. Lett. 82, 4611 (1999).

[15] G. Vemuri, G.S. Agarwal, K.V. Vasavada, Phys. Rev. Lett. 79, 3889 (1997).

[16] A. Rahman, J.H. Eberly, Phys. Rev. A 58, R805 (1998).

[17] D. Bortman-Arbiv, A.D. Wilson-Gordon, H. Friedmann, Phys. Rev. A 63, 043818 (2001).

[18] A. Dogariu, A. Kuzmich, H. Cao, L.J. Wang, Opt. Express 8, 344 (2001).

[19] L.J. Wang, A. Kuzmich, A. Dogarlu, Nature 406, 277 (2000).

[20] A.M. Alhasan, J. Fiutak, J. Mod. Opt. 49, 157 (2002).

[21] A.M. Alhasan, J. Fiutak, Rad. Phys. Chem. 68, 73 (2003).

[22] Y. Zhu, Phys. Rev. A 55, 4568 (1997).

[23] A.M. Alhasan, J. Fiutak, in: IV Workshop on Atomic and Molecular Physics, Ed. J. Heldt, SPIE 5258, 136 (2003).

[24] W. Miklaszewski, J. Fiutak, Z. Phys. B 93, 491 (1994).

[25] G.L. Lamb Jr., D.W. Mc Laughlin, in: Solitons, Eds. R.K. Bullough, P.J. Candrey, Springer, Berlin 1980.

[26] D.J. Kaup, Phys. Rev. A 16, 704 (1977). 